Préface

\title{
La puissance des valeurs
}

Sven Biscop

Les académiques n'ont pas manqué d'inventer toute une gamme de qualifications pour l'Union européenne en tant qu'acteur international. Une "soft power", qui préfère les outils non militaires. Une puissance civile, qui cherche à influencer l'environnement international plutôt que de directement poursuivre ses propres intérêts. Une puissance normative, dont les valeurs sont un modèle pour les autres. Une puissance transformatrice, qui vise la transformation des autres par la promotion de ses valeurs. Une puissance tranquille même, qui renonce à la projection du pouvoir. S'il en est point besoin, ces auteurs proposaient la qualification de puissance positive, dont la stratégie n'est pas dirigée contre les autres puissances, mais orienté vers la réalisation d'objectifs positifs (démocratisation, prospérité économique etc.) afin de prévenir les conflits et de stabiliser l'environnement mondiale.

Plus important que l'adjectif dans toutes ces qualifications est le substantif : l'Union se doit d'être une puissance. On vit dans un monde de plus en plus multipolaire, où l'influence mondiale de plusieurs acteurs s'accroît tous les jours. En plus, ces autres pôles mondiaux, notamment les «BRICs» (le Brésil, la Russie, l’Inde, la Chine), sont des acteurs stratégiques, qui se posent des objectifs à long terme, qui mènent une politique proactive afin de les réaliser, et qui développent les capacités nécessaires. Dans un tel contexte, l'Union n'a pas le choix : si elle veut sauvegarder ses intérêts, elle aussi doit être une puissance qui agit d'une façon stratégique. Si la notion d'intérêt a aujourd'hui plutôt une connotation négative, ceci n'est pas très logique. Chaque acteur cherche à sauvegarder ses intérêts, ce qui en soi n’est ni positif ni négatif ; la façon dont il le fait en revanche peut être jugée. Il ne suffit pas alors d'être un modèle, un pôle d'attraction. L'attractivité seule ne génère pas la puissance - une puissance doit agir.

Mais quelle puissance est-ce que l'Union peut devenir ? Sûrement le but n'est pas de retourner au $19^{\text {ième }}$ siècle, à la compétition entre les grandes puissances ? L’Union ne se doit-elle pas d'être une puissance distinctive ? En effet, l'exercice de son pouvoir et sa politique étrangère sont conditionnés d'une part par son modèle propre et ses propres valeurs et d'autre part par l'environnement international. 
Ce qui rend l'Union distinctive, c'est son modèle social : la combinaison de la démocratie, l'économie de marché et l'intervention gouvernementale, au niveau de l'Union comme au niveau des États-membres, qui doit garantir les règles de la concurrence et la sécurité sociale. Ce modèle de société et les valeurs sur lesquels il est basé constitue un contrat social entre les citoyens européens, qui ont droit à un ensemble de «biens publics », et l'Union et ses États-membres, responsables pour l'accès à ces «biens publics » : la sécurité, la prospérité économique, la liberté politique (la démocratie, le respect des droits de l'homme, l'état de droit) et le bien-être social (l'éducation, la santé, l'environnement). Naturellement, ses valeurs doivent inspirer la politique étrangère comme les politiques internes de l'Union, parce qu'elles représentent le cœur même du projet européen. Le but fondamental de toutes les politiques de l'Union est donc de sauvegarder, de renforcer et d'approfondir même, ce modèle et les valeurs sur lesquels il est basé au bénéfice des citoyens européens.

Quel est maintenant l'environnement dans lequel l'Union agit? Le monde actuel est marqué par la multipolarité, par le nombre croissant d'acteurs disposant d'une influence mondiale, certains presque dans tous les domaines de la politique étrangère, d'autres dans certains champs spécifiques uniquement. Mais en même temps, ces acteurs mondiaux sont aussi de plus en plus interdépendants. Ils se voient tous confrontés aux mêmes défis mondiaux complexes : le changement climatique, l'approvisionnement énergétique, la crise économique et financière. En plus, leurs économies sont de plus en plus étroitement liées l'une à l'autre. Cette condition, Giovanni Grevi l'a baptisée «l'interpolarité », une notion qui ne prend pas seulement en compte la puissance croissante des «BRICs » mais aussi l'interdépendance systémique entre les pôles mondiaux.

Cet environnement représente une opportunité pour l'Union. En effet, cette interdépendance et le besoin de collaborer afin de faire face aux défis mondiaux, favorise la collaboration entre les puissances. Cette collaboration n'est assurément pas garantie mais aucun contexte n'est plus favorable à la création d'un contrat social entre les puissances que le monde actuel. Dans un tel contexte, l'Union a la chance de ne pas devoir se concentrer sur la puissance militaire (même si elle doit continuer le développement et surtout l'intégration des capacités de ses États-membres dans ce domaine, si ce n'est que pour des raisons d'« efficiency»). Elle peut par contre favoriser une stratégie basée sur les valeurs. La meilleure façon de sauvegarder ses intérêts et de renforcer son propre modèle social, c'est de promouvoir au plan mondial les valeurs sur lesquels il est basé. Le but n'est pas que les autres acteurs copient le modèle social de l'Union ; mais les valeurs, même si elles peuvent inspirer plusieurs modèles, qui sont universelles. La meilleure façon de promouvoir la stabilité, c'est de promouvoir l'accès des citoyens aux biens publics essentiels partout dans le monde.

Ceci ne se fera en tout cas pas par la force. Cela ne se fera même pas par les politiques de conditionnalité que l'Union a poursuivies jusqu'ici. Vis-à-vis des autres puissances mondiales, l'Union ne peut essayer que de les convaincre de ses valeurs, par le biais de collaborations concrètes sur des projets spécifiques, inspirés par les intérêts et défis 
communs. Graduellement, via de telles collaborations (contre la piraterie, sur la nonprolifération, sur la bonne gouvernance) l'Union peut espérer socialiser les autres puissances à sa vision d'un «multilatéralisme efficace » et les transformer en puissances responsables, qui contribuent dans l'intérêt de tous à la résolution des problèmes mondiaux.

Il n'y a aucun doute que l'Union a les moyens de devenir une puissance - si seulement elle le voulait. Ceci mène à la question : quelle Europe voulons-nous ? Une Europe qui n'est qu'un marché, ne sera jamais une puissance. Cette «Europe-processus », un forum pour une collaboration fonctionnelle entre États souverains selon les besoins du marché interne, manque le centre de gravité politique pour être un acteur stratégique. Une Europe mondiale ne peut être alors qu'une «Europe-projet » : une Union politique toujours plus étroite, dans laquelle les États-membres mutualisent leur souveraineté à fin de poursuivre avec une efficacité maximale une stratégie commune. 